\title{
Psychological control is a key modulator of fibromyalgia symptoms and comorbidities
}

\section{Katrina Malin Geoffrey Owen Littlejohn}

Departments of Medicine and Rheumatology, Monash University and Monash Medical Centre, Melbourne, Australia
Correspondence: Geoffrey Owen Littlejohn

Monash Rheumatology, Level 3,

Block E, Monash Medical

Centre, 246 Clayton Road,

Clayton, Victoria, Australia 3168

Tel +6I 395943565

Fax +6I 395946512

Email geoff.littlejohn@monash.edu
This article was published in the following Dove Press journal: Journal of Pain Research

I November 2012

Number of times this article has been viewed

Objective: We hypothesized that fibromyalgia (FM) patients would report lower levels of psychological control mechanisms and that higher levels of control would moderate key symptoms associated with FM, such as pain, fatigue, perceived stress, and mood disturbance.

Methods: Ninety-eight women with FM diagnosed according to American College of Rheumatology criteria and 35 matched pain-free women were identified. Applied questionnaires included the Fibromyalgia Impact Questionnaire, Profile of Mood States, Perceived Control of Internal States Scale, Perceived Stress Scale, and Mastery Scale. Differences were sought using $t$-tests, one-way analysis of variance, bivariate correlations, and multiple regression analysis.

Results: Comparison between FM patients and healthy individuals found significant differences in control (Perceived Control of Internal States Scale and Mastery Scale), pain, perceived stress, fatigue, confusion, and mood disturbance (all $P<0.001$ ). There were significant associations found between both high and low levels of control on stress, mood, pain, and fatigue $(P<0.001-0.05)$. Strong negative correlations were present between internal control and perceived stress $(P<0.0005)$.

Conclusion: FM patients use significantly different control styles compared with healthy individuals. Levels and type of psychological control buffer mood, stress, fatigue, and pain in FM. Control appears to be an important "up-stream" process in FM mechanisms and is amenable to intervention.

Keywords: fibromyalgia, psychological, control, mastery, stress

\section{Introduction}

Part of the experience of pain is perception based and influenced by cognitive, behavioral, personality, and learning attributes. An individual's sense of control is an element of perception that has a major influence on pain. The construct of control is complex and multidimensional. ${ }^{1}$ Lack of control has been associated on the one hand with psychological disorders, including anxiety and depression, and on the other with physical illness. ${ }^{2}$ Stress has also been argued to be a product of lack of control. ${ }^{3}$

In general terms, control can be understood as two similarly related constructs. Firstly, it is seen as an internal state that can influence a causal relationship that allows outcomes based on one's own beliefs and actions. Secondly, it is seen as an external state with subsequent outcomes achieved from others, or fate, chance, or luck. ${ }^{4,5}$ Destiny is thus understood to be shaped either by an individual's own ability or by the effects of others. From a health perspective, higher levels of internal control produce more active coping and thus better adjustment to life predicaments. In contrast, having high levels of external control may elicit a more passive coping approach and thus a 
potential for higher levels of disability. ${ }^{6}$ Both external and internal controls exist on a spectrum and are not mutually exclusive. A corollary to these constructs is "perceived control," defined as "the belief that one can determine one's own internal states and behaviors, influence one's environment, and/or bring about their desired change."

In the context of chronic pain, individuals with high internal control mechanisms report more active coping techniques to minimize their pain and also have lower psychological distress. ${ }^{7}$ They function better than those with low internal control who report passive coping techniques. ${ }^{5,8} \mathrm{~A}$ betterfunctioning internal control mechanism associates with a better adjustment to pain and/or other symptoms. In other words, the more one feels in control of a situation, the easier it is to facilitate the adaptation process that is important in managing a chronic health condition. Being in control and being able to adapt to new situations, including chronic pain, is fundamental to the establishment of self-management strategies that have been shown to be effective in chronic pain disorders. ${ }^{9}$

In patients with fibromyalgia (FM), the level of the internal locus of control has been directly linked to the effects of coping with a long-term chronic illness. ${ }^{10}$ Barriers to better control in this context include stress and the negative impact that FM has on everyday life. Women with FM are not only more likely to be vulnerable to the negative effects of stress but have fewer psychological resources, including pain coping techniques, and fewer networks to assist in social support when compared with women with osteoarthritis. ${ }^{11}$

There are few studies that investigate control in FM. FM patients have reported lower control over their pain in comparison with those with rheumatoid arthritis or systemic lupus erythematosus, and treatment that used a self-management approach to increase control showed FM patients to improve significantly. ${ }^{12}$ It has also been shown that those with higher internal control, compared with those with lower control levels, reported greater positive change coming about through different therapeutic interventions. Higher levels of control in FM correlated with increased physical activity levels, improved quality of life, and less pain and fatigue. Of note, in a number of studies, nonpharmacological treatment methods, including self-management approaches, produced more positive outcomes than pharmacologic methods, emphasizing the clinical importance of better understanding of these processes. ${ }^{9,13}$ In contrast, lower levels of internal control in FM patients are associated with higher levels of anxiety and increased pain intensity. ${ }^{14-16}$

The level of control also interacts with other psychological variables. FM patients diagnosed with high levels of depression tended to report a higher external control style, reporting that the FM condition was a result of medical (external) rather than psychological (internal) factors. ${ }^{17,18}$ In contrast, a study investigating the influence of depression on the control (attribution) style in FM found inconclusive results. $^{19}$

The current study is based on the theoretical premise that higher perceived control associates with lower FM-related symptoms. Firstly, we investigated whether the type of control, internal or external, differed between the FM and healthy control (HC) groups. Specifically, we sort whether the FM group, when compared with the HC group, would report lower levels of internal and external control (as measured by the Mastery Scale). Secondly, we studied whether different levels (low, moderate, high) of internal and external control would modulate levels of pain, fatigue, confusion, stress, and mood, and whether significant interactions would be found between FM and HC groups. Next, we explored the relationship between internal control and FM clinical features with the expectation that with increased levels of perceived control and mastery there would be a decrease in perceived stress, pain, fatigue, and mood states. Finally, we examined whether control (internal and external) is a predicator of stress in FM.

\section{Methods}

Ethics approval was obtained through the relevant committees of Monash University and Monash Medical Centre, Melbourne, Australia. The participants consisted of volunteer women who were sourced from a variety of areas, including an FM self-management program, notices in local newspapers, an FM treatment clinic, and local rheumatologists. Ninety-nine female FM patients fulfilling American College of Rheumatology 1990 classification criteria and 36 female $\mathrm{HCs}$, all healthy individuals with no pain condition and recruited by word of mouth, were identified. All participants were sent written information regarding the study, along with a consent form, which, when signed, was followed by a series of questionnaires. These included the Fibromyalgia Impact Questionnaire (FIQ), ${ }^{20}$ Profile of Mood States (POMS), ${ }^{21}$ Perceived Stress Scale (PSS), ${ }^{22}$ Perceived Control of Internal States Scale (PCOISS), ${ }^{1}$ and Mastery Scale. ${ }^{23}$

The details of these constructs are as follows. The FIQ is represented in three sections, which, when combined, produce an overall score. The first section focuses on the person's perception of their ability to perform functional tasks, scoring on a Likert scale with $0=$ always able to do through to $3=$ never able to do. The next section identifies 
two items that focus on how many days the patient (1) felt good and (2) missed work. The final section is a series of visual analog scales ranging from 0 (no issues presented) to 10 (worst possible issues presented), and the patient reports on their ability to work, levels of pain, fatigue, restfulness, stiffness, anxiety, and depression. For this study we examined levels of pain, fatigue, restfulness, anxiety, and depression using the FIQ. The POMS assesses the current mood state through use of a checklist of 65 adjectives. Each adjective was rated on a Likert scale from $1=$ not at all through to 5 = extremely. The factors examined were tension/anxiety, depression/dejection, anger/hostility, fatigue/inertia, vigor/ activity, and confusion/bewilderment. Individual scores represent the level of that particular mood. The PSS is a ten-item scale examining the levels of unpredicted, uncontrollable, or overloaded factors in patients' lives. A 5-point Likert scale is used to rate agreement to the various states from $1=$ never through to $5=$ very often. Higher scores indicate greater perceived distress. The PCOISS measures the degree to which individuals feel they have control of their thoughts, emotions, and physical reactions, which, in turn, moderates the impact of events on their wellbeing. A 5-point Likert scale, rated from $1=$ strongly agree to $5=$ strongly disagree, is used to assess 14 items. High scores indicate a high level of perceived control. The Mastery Scale rates the patients' beliefs in their ability to control situations and to deal with possible difficulties and challenges. A 4-point Likert scale is used, with patients rating their agreement to the states from $1=$ strongly agree to $4=$ strongly disagree to assess seven items. High scores indicate a high level of mastery.

\section{Statistical analysis}

SPSS (PASW version 18, SPSS, Inc, Chicago, IL) was used in the analysis. Initial descriptive analysis was conducted along with normality checks. Means and standard deviations, $t$-tests, analysis of variance, Tukey comparisons, bivariate correlations, and multiple regressions were used to explore the influence of control on symptoms associated with FM.

\section{Results}

Table 1 summarizes the demographics of the two groups. The study consisted of 135 individuals, 99 of whom had FM. There were differences between the groups in regard to a number of sociodemographic factors, including age, education, work status, and income. Some of these factors are a result of FM-related disability and are not present in HCs.

The means, standard deviations, and $t$-tests reported significant differences between the FM and $\mathrm{HC}$ groups for
Table I Demographic variables of FM $(n=98)$ and HC $(n=35)$ participants in the study

\begin{tabular}{|c|c|c|}
\hline & FM (\%) & HC (\%) \\
\hline \multicolumn{3}{|l|}{ Age (years) } \\
\hline $18-29$ & 8.7 & 42.9 \\
\hline $30-39$ & 18.5 & 34.3 \\
\hline $40-49$ & 22.8 & 11.4 \\
\hline $50-59$ & 31.5 & $1 \mathrm{I} .4$ \\
\hline $60-69$ & 18.5 & 0.00 \\
\hline \multicolumn{3}{|l|}{ Marital status } \\
\hline Single & 6.5 & 31.4 \\
\hline Significant relationship & 7.6 & 14.3 \\
\hline Married/de facto & 70.6 & 42.9 \\
\hline Separated & 2.2 & 5.7 \\
\hline Divorced & 13.0 & 5.7 \\
\hline \multicolumn{3}{|l|}{ Education } \\
\hline Secondary & 43.5 & 22.9 \\
\hline Tertiary & 41.4 & 28.6 \\
\hline Higher degree & 14.1 & 48.6 \\
\hline \multicolumn{3}{|l|}{ Work status } \\
\hline Full time & 17.4 & 68.6 \\
\hline Part time & 34.8 & 28.6 \\
\hline Casual & 7.6 & 2.9 \\
\hline \multicolumn{3}{|l|}{ Occupation } \\
\hline Semi-professional & 25.0 & 11.4 \\
\hline Professional & 20.7 & 54.3 \\
\hline Self-employed & 3.3 & 5.7 \\
\hline Retired & 14.1 & 0.00 \\
\hline Unemployed & 3.3 & 0.00 \\
\hline Home/caring & 19.6 & 2.9 \\
\hline Student & 4.3 & 22.9 \\
\hline \multicolumn{3}{|l|}{ Income } \\
\hline$<\$ 20,000$ & 38.6 & 17.1 \\
\hline$\$ 20,000-40,000$ & 33.7 & 25.7 \\
\hline$\$ 41,000-60,000$ & 14.5 & 34.3 \\
\hline$\$ 61,000-80,000$ & 3.6 & 11.4 \\
\hline$+\$ 100,000$ & 6.0 & 0.00 \\
\hline
\end{tabular}

Abbreviations: $\mathrm{FM}$, fibromyalgia; $\mathrm{HC}$, healthy controls.

all domains of FM, with all symptom characteristics being higher in the FM group (Table 2). The levels of anxiety and depression reported by the FM patients were only moderate in severity, whereas sleep and fatigue rated as higher. Fatigue rated higher than pain. Stress was significantly higher in the FM group. Levels of control, both internal and external, were significantly different in the $\mathrm{HC}$ group.

The level of internal or external control was then evaluated in tertiles in order to assess for gradient effects of control on FM clinical features. The relationship between the highest and lowest tertile levels of internal control and the outcomes associated with FM are shown in Table 3. It is noted that as the level of internal control decreased, the level of FM-related symptoms increased. All mean symptom outcomes were significantly different between low and high levels of PCOISS 
Table 2 FM-related characteristics, stress, and control in FM $(\mathrm{n}=98)$ and $\mathrm{HC}(\mathrm{n}=35)$ groups

\begin{tabular}{|c|c|c|c|c|c|}
\hline & \multicolumn{2}{|l|}{$\underline{\mathbf{F M}}$} & \multicolumn{2}{|l|}{$\underline{\mathrm{HC}}$} & \multirow[t]{2}{*}{$P$-value } \\
\hline & Mean & SD & Mean & SD & \\
\hline \multicolumn{6}{|l|}{ Characteristic } \\
\hline Pain (FIQ) & 6.34 & 2.34 & 0.19 & 0.47 & $<0.001$ \\
\hline Fatigue (FIQ) & 7.90 & 2.03 & 2.63 & 2.24 & $<0.000 \mathrm{I}$ \\
\hline Sleep (FIQ) & 7.79 & 2.18 & 3.56 & 2.78 & $<0.0001$ \\
\hline Confusion (POMS) & 10.14 & 4.93 & 6.03 & 5.56 & $<0.000 \mathrm{I}$ \\
\hline Depression (FIQ) & 3.76 & 2.74 & 1.51 & 2.65 & $<0.0001$ \\
\hline Anxiety (FIQ) & 4.48 & 2.83 & 1.94 & 2.30 & $<0.0001$ \\
\hline \multicolumn{6}{|l|}{ Control } \\
\hline Internal (PCOISS) & 57.48 & 9.76 & 63.83 & 10.27 & $<0.05$ \\
\hline External (Mastery Scale) & 16.58 & 3.21 & 18.25 & 3.12 & $<0.05$ \\
\hline \multicolumn{6}{|l|}{ Stress } \\
\hline Perceived stress & 28.96 & 5.65 & 24.69 & 7.28 & $<0.001$ \\
\hline
\end{tabular}

Abbreviations: FIQ, Fibromyalgia Impact Questionnaire; FM, fibromyalgia; HC, healthy controls; PCOISS, Perceived Control of Internal States Scale; POMS, Profile of Mood States; SD, standard deviation.

for both FM and $\mathrm{HC}$, with the exception of pain in the FM group. Thus, high levels of internal control were associated with less sleep disturbance, fatigue, and confusion. However, pain showed a ceiling effect, in that although higher levels of internal control were associated with lower levels of pain, both medium and low levels of internal control made no difference. In contrast, in the HC group, pain was significantly different according to the level of internal control, although absolute pain levels were significantly lower than in the FM group.

The relationship between the highest and lowest tertile levels of external control and the outcomes associated with
FM are shown in Table 4. This shows significant associations between the level of external control and the clinical characteristics of FM in the FM group. Thus, low levels of external control were associated with fewer symptoms, and overall there was a significant difference between the highest and lowest tertile groups. In the HC group the level of external control was associated only with pain, confusion, stress, and depression.

Comparison of the type of control (internal versus external) in FM showed that internal control displayed slightly different responses to external control. Table 5 summarizes this. Both mood and FM symptoms differed significantly between low and high tertile levels, but sleep was different between all three levels, low, medium, and high. External control was significantly different between low to medium and medium to high levels for all FM symptoms, with the exception of pain. Significant stress level differences were reported between all levels of control. Mood and sleep responses reported significant differences between low to high and low to medium levels. Stress responses reported higher sensitivity, with differences found between all levels of external control. These dissimilarities indicate that the two scales do identify different aspects of the construct of control.

It is important to note that mood disturbance in this group was only moderate. For instance, FM patients with the lower levels of internal control still reported only moderate levels of depression (4.58) and anxiety (5.13). Table 6 shows the correlations between both types of control and the associ-

Table 3 Mean scores and ANOVA (F) for highest and lowest tertiles of internal control (PCOISS) for FM and HC groups

\begin{tabular}{|c|c|c|c|c|c|c|c|}
\hline \multirow[t]{3}{*}{ Characteristic } & \multirow[t]{3}{*}{ Group } & \multicolumn{4}{|c|}{ PCOISS level } & \multirow[t]{3}{*}{$\mathbf{F}$} & \multirow{3}{*}{$\begin{array}{l}\text { ANOVA } \\
P \text {-value }\end{array}$} \\
\hline & & \multicolumn{2}{|c|}{ Highest } & \multicolumn{2}{|c|}{ Lowest } & & \\
\hline & & Mean & SD & Mean & SD & & \\
\hline Pain & FM & 5.40 & 2.01 & 6.55 & 2.01 & 2.22 & NS \\
\hline (FIQ) & $\mathrm{HC}$ & 0.00 & 0.00 & 0.50 & 0.84 & 8.16 & $<0.005$ \\
\hline Fatigue & $\mathrm{FM}$ & 6.60 & 2.30 & 8.52 & 1.84 & 6.29 & $<0.005$ \\
\hline (FIQ) & $\mathrm{HC}$ & 2.17 & 1.85 & 4.18 & 2.18 & 4.42 & $<0.05$ \\
\hline Sleep & $\mathrm{FM}$ & 6.40 & 2.28 & 8.48 & 1.69 & 6.15 & $<0.005$ \\
\hline (FIQ) & $\mathrm{HC}$ & 3.07 & 2.46 & 5.00 & 2.83 & 3.27 & $<0.005$ \\
\hline Confusion & $\mathrm{FM}$ & 7.15 & 4.36 & 11.90 & 5.18 & 6.24 & $<0.005$ \\
\hline (POMS) & $\mathrm{HC}$ & 3.21 & 1.85 & 10.82 & 6.85 & 11.35 & $<0.0001$ \\
\hline Stress & $\mathrm{FM}$ & 23.65 & 5.16 & 31.77 & 5.00 & 16.84 & $<0.0001$ \\
\hline (PSS) & $\mathrm{HC}$ & 19.36 & 4.24 & 31.09 & 5.72 & 23.41 & $<0.0001$ \\
\hline Depression & $\mathrm{FM}$ & 2.20 & 2.02 & 4.58 & 3.11 & 5.11 & $<0.005$ \\
\hline (POMS) & $\mathrm{HC}$ & 0.14 & 0.36 & 3.45 & 3.05 & 10.95 & $<0.0001$ \\
\hline Anxiety & $\mathrm{FM}$ & 2.75 & 2.27 & 5.13 & 2.99 & 5.57 & $<0.005$ \\
\hline (POMS) & $\mathrm{HC}$ & 1.14 & 0.36 & 3.10 & 2.39 & 3.92 & $<0.05$ \\
\hline
\end{tabular}

Abbreviations: ANOVA, analysis of variance; FIQ, Fibromyalgia Impact Questionnaire; FM, fibromyalgia; HC, healthy controls; NS, not significant; PCOISS, Perceived Control of Internal States Scale; POMS, Profile of Mood States; PSS, Perceived Stress Scale; SD, standard deviation. 
Table 4 Mean scores and ANOVAs (F) for highest and lowest tertile levels of external control (Mastery Scale) for FM and HC groups. Note that low Mastery Scale level means high external control level

\begin{tabular}{|c|c|c|c|c|c|c|c|}
\hline \multirow[t]{3}{*}{ Characteristic } & \multirow[t]{3}{*}{ Group } & \multicolumn{4}{|c|}{ Mastery level } & \multirow[t]{3}{*}{$\mathbf{F}$} & \multirow{3}{*}{$\begin{array}{l}\text { ANOVA } \\
\text { P-value }\end{array}$} \\
\hline & & \multicolumn{2}{|c|}{ Highest } & \multicolumn{2}{|c|}{ Lowest } & & \\
\hline & & Mean & SD & Mean & SD & & \\
\hline Pain & FM & 5.96 & 2.22 & 7.17 & 2.63 & 2.63 & NS \\
\hline (FIQ) & $\mathrm{HC}$ & 0.08 & 0.29 & 0.75 & 0.96 & 3.90 & $<0.05$ \\
\hline Fatigue & FM & 7.29 & 2.48 & 8.67 & 1.84 & 3.36 & $<0.05$ \\
\hline (FIQ) & $\mathrm{HC}$ & 2.50 & 1.92 & 3.23 & 2.80 & 1.11 & NS \\
\hline Sleep & FM & 7.00 & 2.25 & 8.73 & 1.87 & 5.47 & $<0.01$ \\
\hline (FIQ) & $\mathrm{HC}$ & 3.00 & 2.17 & 4.38 & 3.23 & 1.6 & NS \\
\hline Confusion & $\mathrm{FM}$ & 8.04 & 4.98 & 12.79 & 4.55 & 7.57 & $<0.01$ \\
\hline (POMS) & $\mathrm{HC}$ & 2.92 & 1.78 & 10.00 & 6.73 & 4.79 & $<0.05$ \\
\hline Stress & $\mathrm{FM}$ & 23.91 & 4.90 & 33.10 & 4.45 & 27.38 & $<0.001$ \\
\hline (PSS) & $\mathrm{HC}$ & 18.83 & 4.55 & 29.15 & 6.67 & 13.02 & $<0.001$ \\
\hline Depression & FM & 5.74 & 2.02 & 20.90 & 2.80 & 12.67 & $<0.001$ \\
\hline (POMS) & $\mathrm{HC}$ & 3.45 & 0.79 & 22.75 & 3.47 & 5.31 & $<0.05$ \\
\hline Anxiety & $\mathrm{FM}$ & 8.75 & 2.85 & 15.48 & 2.97 & 5.97 & $<0.01$ \\
\hline (POMS) & $\mathrm{HC}$ & 4.95 & 1.38 & 14.75 & 2.45 & 6.14 & $<0.05$ \\
\hline
\end{tabular}

Abbreviations: ANOVA, analysis of variance; FIQ, Fibromyalgia Impact Questionnaire; FM, fibromyalgia; HC, healthy controls; POMS, Profile of Mood States; PSS, Perceived Stress Scale; SD, standard deviation.

ated clinical characteristics of FM in both groups. Inverse relationships were found with higher $r$-values for the $\mathrm{HC}$ group compared with the FM group.

Regression analysis was conducted within the FM population in order to explore whether internal and/or external control would predict outcomes of stress and other selected components of the FM phenotype (see Table 7). It was found that internal and external control together was the best predictor of stress, with $53 \%$ of the variance explained by this combination. In regards to stress, external control held higher contribution to this model with $48.5 \%$, whereas internal control contributed 32\%. Internal and external control contributed less significantly to confusion, anxiety, and depression and not at all to pain and fatigue. It appears

Table 5 Significant differences between tertile groups of internal control and external control and clinical features of FMa

\begin{tabular}{|c|c|c|c|c|}
\hline \multirow[t]{2}{*}{ Characteristics } & \multicolumn{2}{|c|}{$\begin{array}{l}\text { Internal control } \\
\text { (PCOISS) }\end{array}$} & \multicolumn{2}{|c|}{$\begin{array}{l}\text { External control } \\
\text { (Mastery Scale) }\end{array}$} \\
\hline & FM & $\mathrm{HC}$ & FM & $\mathrm{HC}$ \\
\hline Pain & NS & $\mid-3 / I-2$ & NS & $I-3 / I-2$ \\
\hline Fatigue & $1-3$ & $1-3$ & $\mathrm{I}-3$ & NS \\
\hline Sleep & $|-3 /|-2$ & $\mathrm{I}-3$ & $|-3 /|-2$ & NS \\
\hline Confusion & $1-3$ & $\mathrm{I}-3$ & $\mathrm{I}-3$ & $|-3 /|-2$ \\
\hline Stress & $1-3$ & $1-3 / 2-3$ & $|-3 /|-2 / 2-3$ & $|-3 /|-2$ \\
\hline Anxiety & $1-3$ & $1-3$ & $|-3 /|-2$ & NS \\
\hline Depression & $1-3$ & $\mathrm{I}-3$ & $|-3 /|-2$ & $\mathrm{I}-3$ \\
\hline
\end{tabular}

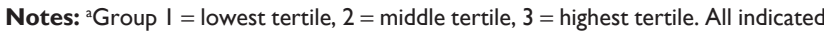
grouping significant at $P<0.05$.

Abbreviations: FM, fibromyalgia; HC, healthy controls; NS, not significant; PCOISS, Perceived Control of Internal States Scale. that control mechanisms contribute significantly to stress levels in FM.

\section{Discussion}

Control has been seen primarily as a means of understanding how aversive predicaments are managed. Control was a reaction to a predicament. However, the concept of control has expanded to include how cognitions, emotions, and behaviors each interact with control and hence influence outcomes. Part of this adaptive process reflects elements of personality by use of "stable traits" and their influence on one's ability to adapt to change and, as such, their effect on one's status of health behavior. ${ }^{4}$ This is relevant to chronic pain states. It has been found that control has a close association with

Table 6 Correlations between highest and lowest tertiles of internal control and external control and clinical features of FMa

\begin{tabular}{llllll}
\hline Characteristics & \multicolumn{2}{l}{$\begin{array}{l}\text { Internal control } \\
\text { (PCOISS) }\end{array}$} & & \multicolumn{2}{l}{$\begin{array}{l}\text { External control } \\
\text { (Mastery Scale) }\end{array}$} \\
\cline { 2 - 3 } \cline { 6 - 7 } & FM & HC & & FM & HC \\
\hline Pain & NS & $-0.640^{* *}$ & & -0.245 & $-0.450^{* *}$ \\
Fatigue & $-0.340^{* *}$ & $-0.452^{* *}$ & & $-0.347^{* *}$ & NS \\
Sleep & $-0.395^{* *}$ & $-0.399^{* *}$ & & $-0.390^{* *}$ & NS \\
Confusion & $-0.618^{* *}$ & $-0.770^{* *}$ & $-0.679 * *$ & $-0.741^{* *}$ \\
Stress & $-0.453^{* *}$ & $-0.577^{* *}$ & $-0.443^{* *}$ & $-0.604^{* *}$ \\
Anxiety & $-0.411^{* *}$ & $-0.416^{* *}$ & $-0.414^{* *}$ & $-0.454^{*}$ \\
Depression & $-0.451^{* *}$ & $-0.610^{* *}$ & & $-0.510^{* *}$ & $-0.583^{* *}$ \\
\hline
\end{tabular}

Notes: ${ }^{a}$ Correlations expressed as $r$-values between highest and lowest tertiles; $* P<0.05 ; * * p<0.0001$.

Abbreviations: FM, fibromyalgia; HC, healthy controls; NS, not significant; PCOISS, Perceived Control of Internal States Scale. 
Table 7 Summary of significant multiple regression analyses of control in predicting characteristics associated with fibromyalgia

\begin{tabular}{|c|c|c|c|c|c|}
\hline Analysis no & Dependent & Adj $R^{2}$ & $\mathbf{R}^{2}$ & Independent & Standardized $\beta$ \\
\hline \multirow[t]{2}{*}{1} & Perceived stress & 0.51 & 0.53 & PCOISS & $0.322 * *$ \\
\hline & $F(2,79)=43.82, P<0.000$ & & & Mastery Scale & $0.485^{* * *}$ \\
\hline \multirow[t]{2}{*}{2} & Confusion & 0.23 & 0.25 & PCOISS & $0.291 *$ \\
\hline & $F(2,79)=13.08, P<0.000$ & & & Mastery Scale & $0.264 *$ \\
\hline \multirow[t]{2}{*}{3} & Anxiety & 0.19 & 0.23 & PCOISS & $-0.252 *$ \\
\hline & $F(2,79)=10.56, P<0.05$ & & & Mastery Scale & $-0.259 *$ \\
\hline \multirow[t]{2}{*}{4} & Depression & 0.27 & 0.29 & PCOISS & NS \\
\hline & $F(2,79)=16.20, P<0.000$ & & & Mastery Scale & $-0.374 * *$ \\
\hline
\end{tabular}

Notes: $* P<0.05 ; * * p<0.01$; ***p $<0.001$.

Abbreviations: NS, not significant; PCOISS, Perceived Control of Internal States Scale.

functional capacity, where stronger internal beliefs of control align with higher success rates with treatments. ${ }^{13}$ Individuals with a higher sense of internal control proactively seek and use strategies that result in fewer subsequent limitations. In contrast, in relation to chronic pain, patients who perceive that outcomes are controlled by chance (ie, those with high levels of external control and hence low levels of mastery), report higher helplessness scores and that their coping techniques are not effective in adjusting or decreasing their pain. ${ }^{5,16}$ Thus, internal and external control processes each seem able to contribute to or modulate clinical outcomes in patients with chronic pain.

Control, in general, links beliefs, feelings, and behaviors to predictable outcomes. Some consider fate or chance to represent an external state that has no effect on the ability of the individual's beliefs, feelings, or actions to shape consequences and outcomes. ${ }^{14}$ Although studies reviewing the use of control within the FM population are limited, one study of FM patients reported use of external control mechanisms in comparison with patients with rheumatoid arthritis. ${ }^{24}$ Another study in women with FM found an association between external control and depressed mood, suggesting that increased external control was a strong predictor of depression. ${ }^{7}$ It has been shown that decreasing a sense of external control in FM will associate with significantly better outcomes in a multidisciplinary program. ${ }^{9}$

In this study we sought to further examine aspects of control in FM. We were interested to see where control, both internal and external, was placed in the various elements that characterize this common pain disorder. We identified patients with the characteristic clinical phenotype of FM based on established classification criteria. Our healthy pain-free controls were moderately well matched, with some demographic differences between the FM and $\mathrm{HC}$ groups. However, the subjects appear representative of other studies with university educated, married women between the ages of 50 and 55 years who are working and/ or housewives. ${ }^{25,26}$ It has been shown that socioeconomic factors such as age, education, work status, and income may all affect the expression of pain, fatigue, poor sleep, stress, and other related variables. ${ }^{27,28}$ These factors may have influenced the changes seen in the FM group compared with the HC group. However, our FM population showed typical levels of clinical features as found in other studies. Fatigue and sleep disturbance rated higher than pain, as is often seen. Cognitive disturbance (as measured by confusion from the POMS instrument) was high. These characteristics shape the FM phenotype. ${ }^{29}$ In addition, our patients had, on average, moderate levels of anxiety and depression and moderate to high self-rated psychological distress. The internal and external control scales were both significantly lower in FM patients compared with the healthy controls, as was hypothesized.

The FM patient population showed a gradient effect between FM characteristics and comorbidities and both types of control - lower internal control and higher external control - each associated with increased FM symptoms, stress, and mood changes. Of the FM characteristics of pain, fatigue, sleep satisfaction, and confusion, it is noted that only pain did not show a gradient effect between low, medium, and high levels of control, either internal or external. It was felt that this might relate to a ceiling effect of pain, in that all patients had pain as a prerequisite entry criterion into the study. Thus, although pain levels differed significantly between the FM group and the HC group, there was no buffering effect toward pain levels from within the FM group.

Internal control and external control were further entered in multiple regression analysis together with FM-related factors, including confusion, anxiety, depression, stress, pain, 
and fatigue, in order to estimate symptom severity and coping skills (Table 7). Control mechanisms turned out to be the most powerful predictor for stress, with the Mastery Scale explaining $32 \%$ of the variance and $28.5 \%$ for internal control. The effect of stress may be relevant to the FM process. Patients with poor internal control mechanisms may generate more stress, which, in turn, may amplify the FM process. Those placed in situations where there is high external control may also generate similar stress-related outcomes. It has been previously noted that women with FM and a higher external control level than a healthy normal comparator group showed higher anxiety and depression scores. ${ }^{7}$

In our study, it is noted that the healthy controls also showed a significant relationship between different levels of internal control and pain, fatigue, sleep satisfaction, and confusion, as well as stress and mood changes. This suggests that internal control links with all these characteristics in normal healthy people, albeit at much lower levels than in FM patients. The effect of internal control on FM clinical features may be one end of a spectrum that includes normal healthy people at one end and the FM phenotype at the other. Different levels of external control in normal healthy people showed similar associations except for fatigue, sleep satisfaction, and anxiety. The effects of external control factors thus may be more complex on the individual than those that relate to internal control.

There are conceptual differences between the instruments used to assess "internal" and "external" control. Although both capture similar essential elements relating to control, the construct of the internal and external statements differs in important ways. The internal control items as used in the PCOISS instrument target the thoughts associated with control and include: "I find it hard to stop thinking about my problems.", "I realise I am thinking silly thoughts; I can usually stop myself.", "I can usually talk myself out of feeling bad.", "I usually keep my thoughts under control.", and "I have a number of techniques or tricks that I use to stay calm and relaxed." In contrast, the external control items, as used in the Mastery Scale, target more general concepts of control external to the individual, such as: "Whatever happens to me in the future mostly depends on me.", "Sometimes I feel I am being pushed around in life.", "I can do just about anything I really set my mind to.", and "I often feel helpless in dealing with the problems of life." These scales provide for a spectrum of responses and are not mutually exclusive, but they do attempt to target different aspects of control. The intrinsic scale differences therefore give some explanation as to the differences found in Table 6. Overall, there were similar findings between both scales and FM characteristics, in that both lower internal control and higher external control (ie, lower mastery) were associated with higher symptoms of FM and also higher stress in FM patients.

The issue of control is an important aspect in the picture of the FM individual, as living with a chronic condition involves adaptation. The ability to control internal and behavioral responses that shape the desired outcome links more closely to adjustment based on the control over the emotional response rather than the situation itself. ${ }^{1}$ Thus, the predictability over these internal states (internal control) can be a useful tool for self-management, as it relates to the behavioral intention of a patient to engage in change, eg, in physical activity. A person who believes they can cope with the pain of FM will engage in more physical activity than a person who does not. ${ }^{13}$ Combining this with the individual's belief system produces a blueprint of how the FM patient copes with relevant symptoms.

Various studies in FM populations have explored a person's internal states, particularly how thoughts, emotions, and physical reactions might influence outcomes. This is pertinent to the central sensitization process and exacerbation of persistent pain. ${ }^{30}$ Patients instructed in methods to improve their perceived locus of control have shown changes in their subsequent functional magnetic resonance imaging (fMRI) scan. ${ }^{31,32}$ Before instruction, the pain-related fMRI changes are typical of those seen in FM. After instruction in methods to improve internal control, the fMRI reflects less of the FM changes, and the patients report fewer FM symptoms. This infers that control is an important "up-stream" mediator in the psychological processes that associate with FM clinical features.

Limited conclusions can be drawn from this study, with only cross-sectional and exploratory outcomes reported here, and thus further investigations require a longitudinal focus to replicate the findings. It is unclear from this cross-sectional study as to whether changes in control are a reaction to the clinical condition of FM or whether they are intrinsic to the process causing the altered biology that result in typical features. In addition, we do not report the duration of pain, which means that drawing inferences about the pain is limited. It has been well documented that the individuals who are often found within studies such as these are individuals who are well entrenched in a difficult mix of chronic feedback loops of psychological and 
behavioral responses. Hence, we used intragroup comparisons and sought gradient effects using tertile comparisons to enhance the results in our FM cohort. We also recognize that the various patient-reported outcomes that we used are each measuring overlapping constructs that indirectly and somewhat artificially sample different aspects of a complex psychological process.

Understanding the impact of control and its relationship to the development and exacerbation of FM has major implications for treatment. It has been established that a self-management approach is an effective way to improve outcomes in FM. Incorporating techniques that bring change to an individual's belief that they can influence or control their internal states and hence their reactions to life predicaments is a cost-effective and sustainable strategy. Improvement in control needs to be considered as part of FM management.

The ability to control thoughts, emotions, and physical reactions differs significantly between FM and healthy comparators and buffers mood, stress, fatigue, and pain. Control appears an important "up-stream" process in FM mechanisms and outcomes amenable to positive intervention. As control influences healthy behaviors, the application of self-management principles, and the biopsychosocial factors that contribute to the development and exacerbation of central sensitization, it is a key factor in FM that warrants further research.

\section{Disclosure}

The authors report no conflicts of interest in this work.

\section{References}

1. Pallant J. Development and validation of a scale to measure perceived control of internal states. J Pers Assess. 2000;75(2):308-337.

2. Roddenberry A, Renk K. Locus of control and self-efficacy: potential mediators of stress, illness and utilization of health services in college students. Child Psychiatry Hum Dev. 2010;41:353-370.

3. Shapiro D, Schwartz C, Astin J. Controlling ourselves, controlling our world. Am Psychol. 1996;51:1213-1230.

4. Bates M, Rankin-Hill L. Control, culture and chronic pain. Soc Sci Med. 1994;39(5):629-645.

5. Harkapaa K, Jarvikoski A, Mellin G, Hurri H, Lucoma J. Health locus of control beliefs and psychological distress as predictors for treatment outcome in low back pain patients: result of a 3 month follow up of a controlled intervention. Pain. 1991;46:35-41.

6. Wallston M, Wallston B, Smith S, Dobbins C. Perceived control and health. Current Psychological Research and Reviews. 1987;6: $5-25$.

7. Shuster J, McCormack J, Pillai Riddell R, Toplak ME. Understanding the psychosocial profile of women with fibromyalgia syndrome. Pain Res Manag. 2009;14(3):239-245.

8. Turner J, Clancy S. Strategies for coping with low back pain: relationship to pain and disabilities. Pain. 1986;24:355-364.
9. Torres X, Collado A, Arias A, et al. Pain locus of control predicts return to work among Spanish fibromyalgia patients after completion of a multidisciplinary pain program. Gen Hosp Psychiatry. 2009;31(2): $137-145$.

10. Malt EA, Olafsson S, Lund A, Ursin H. Factors explaining variance in perceived pain in women with fibromyalgia. BMC Musculoskelet Disord. 2002;3:12.

11. Davis MC, Zautra AJ, Reich JW. Vulnerability to stress among women in chronic pain from fibromyalgia and osteoarthritis. Ann Behav Med. 2001;23(3):215-226.

12. Burckhardt CS, Bjelle A. Perceived control: a comparison of women with fibromyalgia, rheumatoid arthritis, and systemic lupus erythematosus using a Swedish version of the Rheumatology Attitudes Index. Scand J Rheumatol. 1996;25(5):300-306.

13. Culos-Reed SN, Brawley LR. Fibromyalgia, physical activity, and daily functioning: the importance of efficacy and health-related quality of life. Arthritis Care Res. 2000;13(6):343-351.

14. Gustafsson M, Gaston-Johansson F. Pain intensity and health locus of control: a comparison of patients with fibromyalgia syndrome and rheumatoid arthritis. Patient Educ Couns. 1996;29(2): 179-188.

15. Toomey T, Mann J, Abashian S, Tompson-Pope S. Relationship between perceived self-control of pain. Pain description and function. Pain. 1991;45:129-133.

16. Pellino T, Oberst M. Percpetion of control and appraisal of illness in chronic low back pain. Orthop Nurs. 1992;11:22-26.

17. Brosschot JF, Aarsse HR. Restricted emotional processing and somatic attribution in fibromyalgia. Int J Psychiatry Med. 2001;31(2): 127-146.

18. Goldenberg DL. Fibromyalgia syndrome a decade later: what have we learned? Arch Intern Med. 1999;159(8):777-785.

19. Michielsen HJ, Van Houdenhove B, Leirs I, Vandenbroeck A, Onghena P. Depression, attribution style and self-esteem in chronic fatigue syndrome and fibromyalgia patients: is there a link? Clin Rheumatol. 2006;25(2):183-188.

20. Burckhardt CS, Clark SR, Bennett RM. The fibromyalgia impact questionnaire: development and validation. $J$ Rheumatol. 1991;18(5):728-733.

21. McNair D, Lorr M, Droppleman LF. EdITS manual for the profile of mood states. San Diego, CA: Educational and Industrial Testing Service; 1992.

22. Cohen S, Kamararck T, Mermelstein R. A global measure of percieved stress. J Health Soc Behav. 1983;2:385-396.

23. Pearlin L, Schooler C. The structure of coping. J Health Soc Behav. 1978;19:2-21.

24. Franks H, Cronan T, Oliver K. Social support in women with fibromyalgia: is quality more important than quanity? J Community Psychol. 2004;32:425-438.

25. Boyer AL, Mira Pastor MA, Calatayud NP, Lopez-Roig S, Cantero Terol MC. Comparing fibromyalgia patients from primary care and rheumatology settings: clinical and psychosocial features. Rheumatol Int. 2009;29(10):1151-1160.

26. Walen HR, Oliver K, Groessl E, Cronan TA, Rodriguez V. Traumatic events, health outcomes and heath care use in patients with fibromyalgia. J Musculoskelet Pain. 2001;9(2):19-38.

27. Macfarlane GJ, Norrie G, Atherton K, Power C, Jones GT. The influence of socioeconomic status on the reporting of regional and widespread musculoskeletal pain: results from the 1958 British Birth Cohort Study. Ann Rheum Dis. 2009;68(10):1591-1595.

28. Assumpcao A, Cavalcante AB, Capela CE, et al. Prevalence of fibromyalgia in a low socioeconomic status population. BMC Musculoskelet Disord. 2009;10:64.

29. Wolfe F, Clauw DJ, Fitzcharles MA, et al. The American College of Rheumatology preliminary diagnostic criteria for fibromyalgia and measurement of symptom severity. Arthritis Care Res. 2010;62(5): 600-610. 
30. Jensen MP, Turner JA, Romano JM, Lawler BK. Relationship of painspecific beliefs to chronic pain adjustment. Pain. 1994;57:301-309.

31. Williams D, Ambrose D, Skalski K, Muroff L, Zwink L, Clauw D. Improving internal locus of pain control in fibromyalgia. Arthritis Rheum. 2009;60(10):1418.
32. Williams D, Harris R, Bhavsar R, Clauw D, Zubieta J, Gracely R. Neurocortical representation of locus of control in individuals with fibromyalgia. Arthritis Rheum. 2009;60(S10):1996.

\section{Publish your work in this journal}

The Journal of Pain Research is an international, peer-reviewed, open access, online journal that welcomes laboratory and clinical findings in the fields of pain research and the prevention and managemen of pain. Original research, reviews, symposium reports, hypothesis formation and commentaries are all considered for publication.

\section{Dovepress}

The manuscript management system is completely online and includes a very quick and fair peer-review system, which is all easy to use. Visit http://www.dovepress.com/testimonials.php to read real quotes from published authors. 